\title{
miR-101a and miR-30b contribute to inflammatory cytokine-mediated $\beta$-cell dysfunction
}

\author{
Ying Zheng ${ }^{1}$, Zhen Wang ${ }^{2}$, Yiting Tu², Hongwei Shen ${ }^{1}$, Zhijie Dai ${ }^{2}$, Jian Lin² and Zhiguang Zhou ${ }^{2}$
}

Inflammatory cytokines have a critical role in the progressive deterioration of pancreatic $\beta$-cell function and development of type 1 diabetes. Prolonged exposure of $\beta$-cells to inflammatory cytokines results in gene expression modifications, leading to loss of $\beta$-cell function. MicroRNAs (miRNAs) are small non-coding RNAs acting as key regulators of gene expression. Here, we demonstrate that miR-101a and miR-30b are key players in cytokine-mediated $\beta$-cell dysfunction. We found that IL-1 $\beta$ induces an increase in miR-101a and miR-30b in MIN6 cells, and that the two miRNAs participate in $\beta$-cell dysfunction, including decreased insulin content, gene expression, and increased $\beta$-cell death. miR-101a and miR-30b reduce proinsulin expression and insulin content by directly targeting the transcriptional factor Neurod1. In addition, $\beta$-cell apoptosis mediated by miR-101a and miR-30b is associated with diminished expression level of the antiapoptotic protein Bcl2. Moreover, we show that miR-101a causes an impairment in glucose-induced insulin secretion by decreasing the expression of the transcription factor Onecut2. Taken together, our findings suggest that changes in the levels of miR-101a and miR-30b contribute to cytokine-mediated $\beta$-cell dysfunction occurring during the development and progression of type 1 diabetes.

Laboratory Investigation (2015) 95, 1387-1397; doi:10.1038/labinvest.2015.112; published online 14 September 2015

Type 1 diabetes is a chronic autoimmune disease characterized by an inflammatory response against pancreatic islets, leading to selective and progressive destruction of pancreatic $\beta$-cells. ${ }^{1,2}$ Proinflammatory cytokines such as interleukin (IL)- $1 \beta$, tumor necrosis factor (TNF)- $\alpha$, and interferon (IFN) $-\gamma$, released by infiltrating immune cells, have a vital role in $\beta$-cell failure and the development of type 1 diabetes. ${ }^{1-4}$ Chronic exposure of $\beta$-cells to these cytokines results in impaired insulin biosynthesis and secretion and in $\beta$-cell loss. Thus, it is important to clarify the exact mechanisms by which $\beta$-cells are destroyed, so as to determine the causes of type 1 diabetes and develop new approaches for the treatment of the disease. Several mechanisms have been reported to be involved in the cytotoxic effects of inflammatory cytokines, including the induction of modification in gene expression of pancreas-specific transcription factors and the impairment of key signaling pathways. ${ }^{2,4}$

MicroRNAs (miRNAs) are endogenous small non-coding RNAs that act as post-translational regulators of gene expression. ${ }^{5}$ miRNAs regulate gene expressions mainly by binding to specific sequences of $3^{\prime}$-untranslated regions (UTRs) of target mRNAs, leading to translational repression or mRNA degradation. ${ }^{5,6}$ These molecules are implicated in many physiological and pathological processes. ${ }^{7}$ miRNAs are also involved in the development of diabetes, and have been shown to have potential roles in the regulation of $\beta$-cell functions. ${ }^{8-11}$ miR-375 negatively regulates insulin gene expression by targeting PDK1, and glucose-induced reduction of miR-375 ultimately leads to defective insulin synthesis. ${ }^{8,12}$ The development of diabetes in diabetic $\mathrm{db} / \mathrm{db}$ mice is associated with a strong induction of miR-34 in $\beta$-cells, which reduces glucose-stimulated insulin secretion (GSIS) and affects cell survival, possibly via its target protein Vamp2 and Bcl2. ${ }^{13}$ Recent studies have shown that several miRNAs contribute to the onset and development of type 1 diabetes and have a fundamental role in $\beta$-cell destruction induced by inflammatory cytokines. ${ }^{14,15}$ High levels of miR-21, miR-29, miR-34, and miR-146 are observed in the pancreatic islets of prediabetic NOD mice, a known type 1 diabetes model. Prolonged exposure of MIN6 cells or mouse pancreatic $\beta$-cells to inflammatory cytokines induces marked upregulation of miR-21, miR-29, miR-34a, and miR-146a, which can alter insulin synthesis and secretion and promote $\beta$-cell apoptosis. ${ }^{14,15}$

\footnotetext{
${ }^{1}$ Center for Medical Research, 2nd Xiangya Hospital, Central South University, Changsha, Hunan, China and ${ }^{2}$ Diabetes Center, 2nd Xiangya Hospital, and Institute of Metabolism and Endocrinology, Key Laboratory of Diabetes Immunology, Ministry of Education, Central South University, Changsha, Hunan, China

Correspondence: Dr Z Zhou, PhD, MD, Diabetes Center, 2nd Xiangya Hospital, and Institute of Metabolism and Endocrinology, Key Laboratory of Diabetes Immunology, Ministry of Education, Central South University, 139 Renmin Middle Rd, Changsha, Hunan 410011, China.

E-mail: zhouzg@hotmail.com

Received 12 February 2015; revised 18 June 2015; accepted 6 July 2015
} 
Roggli E et al. ${ }^{14}$ took advantage of global microarray profiling to detect changes in miRNA expression in $\beta$-cells exposed to cytokines, and further determined possible functions of some miRNAs such as miR-21, miR-34a, and miR-146a. Our group chose miRNAs that varied in levels of expression between the microarray assays (including 10 upregulated miRNAs and 10 downregulated miRNAs, for which expression levels were highly consistent in Roggli E's two duplicate microarrays). They were used for further confirmation by real-time PCR analysis, among which miR-101a and miR-30b were induced by proinflammatory cytokines. In this study, we describe the effects of miR-101a and miR-30b on the secretory capacities of $\beta$-cells and their sensitivity to apoptosis and further investigated their possible mechanisms.

\section{MATERIALS AND METHODS Cell Culture and Chemicals}

The insulin-secreting cell line MIN6 was maintained in Dulbecco's modified Eagle's medium (DMEM, Invitrogen, Carlsbad, CA, USA) supplemented with $15 \%$ heat-inactivated fetal calf serum (FBS), $50 \mathrm{IU} / \mathrm{ml}$ penicillin, $50 \mathrm{mg} / \mathrm{ml}$ streptomycin, and $70 \mathrm{mmol} / \mathrm{l} \beta$-mercaptoethanol (Millipore, Billerica, MA, USA). The cells were cultured at $37^{\circ} \mathrm{C}$ in a humidified atmosphere of $5 \% \mathrm{CO}_{2}$. The human embryonic kidney (HEK) 293 cells were cultured in DMEM supplemented with $10 \%$ FBS. Recombinant mouse IL- $1 \beta$ and TNF- $\alpha$ were purchased from Miltenyi Biotec (Teterow, Germany), and IFN- $\gamma$ was obtained from Biolegend (San Diego, CA, USA). Hoechst dye 33342 was purchased from Beyotime (Jiangsu, China).

\section{Overexpression and Inhibition of miRNAs}

To overexpress miRNAs, the cells were transfected with RNA duplexes corresponding with the mature sequence of mmu-miR-101a and mmu-miR-30b (miR-101a mimics and miR-30b mimics, GenePharma, Shanghai, China). An unrelated known miRNA was used as a control (NC mimics). Endogenous activity of the miRNAs was blocked by using the corresponding clear-MiR miRNA inhibitor (GenePharma). The transfections of MIN6 cells were performed with Lipofectamine 2000 (Invitrogen) following the manufacturer's instructions.

\section{miRNA Detection}

The MIN6 cells were treated with either $10 \mathrm{ng} / \mathrm{ml} \mathrm{IL}-1 \beta$, $10 \mathrm{ng} / \mathrm{ml} \mathrm{IFN-} \gamma, 10 \mathrm{ng} / \mathrm{ml} \mathrm{TNF}-\alpha$, or a mix of $10 \mathrm{ng} / \mathrm{ml} \mathrm{IL-} \beta \beta$, $10 \mathrm{ng} / \mathrm{ml} \mathrm{TNF}-\alpha$, and $10 \mathrm{ng} / \mathrm{ml} \mathrm{IFN-} \gamma$ for $24 \mathrm{~h}$. Then, total RNA from the cells was isolated using Trizol (Invitrogen). Mature miRNAs measurements were performed using a quantitative miRNA Detection kit (GenePharma). The results were normalized to the relative expression of U6.

\section{Analysis of the Expression of Protein-Coding Genes}

Total RNA from the cells was extracted using Trizol (Invitrogen), and cDNA was synthesized from $1 \mu \mathrm{g}$ of total
RNA by means of the reverse reaction kit, according to the manufacturer's protocols (Promega, Madison, WI, USA). And then quantitative real-time RT-PCR analysis of proinsulin, Neurod1, Onecut2, and Bcl2 was performed in an iQ5 qRT-PCR detection system (Bio-Rad, Hercules, CA, USA). GAPDH was used as a reference gene for normalization. Primer sequences for Q-PCR: proinsulin forward 5'-TGGC TTCTTCTACACACC CA-3', reverse 5'-TCTAGTTGCAGTA GTTCTCCA-3'; Neurod1 forward 5'-GCCCTTTTGCTAA GA TTGCTGC-3', reverse 5'-GTTTCTTCCAAAGGCAGTA ACGACA-3'; Onecut2 forward 5'-GCCACGCCGCTGGG CAAC-3', reverse 5'-CAGCTCCCGGACGTGGC-3'; Bcl2 forward 5'-TCGCAGAGA TGTCCAGTCAG-3', reverse 5'ATGCCGGTTCAGGTACTCAG-3' GAPDH forward 5'-TCT GAC GTGCCGCCTGGAGA-3', reverse 5'-CAGCCCCGGCA TCGAAGGTG-3'. The qRT-PCR experiments were repeated three times.

\section{Insulin Determination}

The MIN6 cells were transfected with miRNAs mimics or inhibitor. After $48 \mathrm{~h}$, the cells were washed and preincubated for $30 \mathrm{~min}$ in Krebs buffer $(127 \mathrm{mmol} / \mathrm{l} \mathrm{NaCl}, 4.7 \mathrm{mmol} / \mathrm{l}$ $\mathrm{KCl}, \quad 1 \mathrm{mmol} / \mathrm{l} \quad \mathrm{CaCl}_{2}, \quad 1.2 \mathrm{mmol} / \mathrm{l} \quad \mathrm{KH}_{2} \mathrm{PO}_{4}, 1.2 \mathrm{mmol} / \mathrm{l}$ $\mathrm{MgSO}_{4}, 5 \mathrm{mmol} / \mathrm{l} \quad \mathrm{NaHCO}_{3}, \quad 0.1 \% \mathrm{BSA}$, and $25 \mathrm{mmol} / \mathrm{l}$ HEPES, pH 7.4) containing $2 \mathrm{mmol} / \mathrm{l}$ glucose. The medium was discarded, and the cells were incubated in the same buffer. For GSIS, the cells were incubated in a Krebs buffer containing $20 \mathrm{mmol} / \mathrm{l}$ glucose. After $1 \mathrm{~h}$ stimulation, supernatants were collected. And the cells were also lysed in $75 \%$ ethanol and $0.55 \% \mathrm{HCl}$ to evaluate total insulin content. The amount of insulin in the samples was determined by ELISA.

\section{Luciferase Assay}

To generate luciferase reporter constructs, the $3^{\prime}$-UTR regions of Neurod1, Onecut2, or Bcl2 containing the predicted target sites of miR-101a or miR-30b were cloned into pMIRREPORT miRNA expression reporter vector (Ambion, Shanghai, China) by using synthesized fragments. The corresponding mutant plasmids were also constructed. For luciferase reporter assays, pMIR-Neurod1 (Onecut2 or Bcl2)-3'-UTR (WT or MUT) plasmid was co-transfected with miR-101a or miR-30b mimics into the HEK293 cells. The pMIR-REPORT- $\beta$ -gal control vector was co-transfected as an internal control. The cells were collected $48 \mathrm{~h}$ after transfection and detected using the Dual-Luciferase Reporter Assay System (Promega).

\section{Western Blots}

Cells were lysed using SDS lysis buffer containing protease inhibitors. Samples were loaded to 12\% SDS/PAGE gels and subjected to electrophoresis. Proteins were transferred to nitrocellulosemembranes and subsequently determined with antibody against Neurod1 (Epitomics, Burlingame, CA, USA), Bcl2 (Sigma-Aldrich, St Louis, MO, USA), Onecut2 (Santa Cruz Biotechnology, Santa Cruz, CA, USA) and GAPDH (Millipore). 


\section{Cell Death}

For the assessment of cell death, MIN6 cells were plated in 24-well plate and transiently transfected with miRNAs mimics or inhibitor. After 3 days, the cells were stained with Hochest33342, and fraction of dying cells was determined by scoring the cells displaying picnotic nuclei upon the staining. The experiment was carried out blindly, and at least 500 cells per condition were analyzed. Transfection experiments were performed in duplicates and repeated at least thrice in independent experiments.

\section{Statistical Analysis}

All statistical analyses were performed using the SPSS 13.0 software (Chicago, IL, USA). Results are expressed as mean \pm s.d. from at least three separate experiments. Data were analyzed by ANOVA followed by the post hoc Dunnett's test for multiple comparisons. In all cases, $P<0.05$ was considered with statistical significance.

\section{RESULTS}

\section{Determination of Mouse miRNAs That Are Induced by} Inflammatory Cytokines in Pancreatic $\boldsymbol{\beta}$-cells

Roggli et al. ${ }^{14}$ took advantage of miRNA chip analysis to select candidate miRNAs that are involved in cytokine-induced $\beta$-cell failure. To find functional miRNAs and investigate the effects of these miRNAs on the $\beta$-cell function, we first confirmed the changes in expression of selected miRNAs in cytokine-treated $\beta$-cells. The mouse insulin-secreting cell line MIN6 cells were incubated for $24 \mathrm{~h}$ in the presence of a mix of $10 \mathrm{ng} / \mathrm{ml} \mathrm{IL-} 1 \beta, 10 \mathrm{ng} / \mathrm{ml}$ TNF- $\alpha$, and $10 \mathrm{ng} / \mathrm{ml} \mathrm{IFN}-\gamma$, and then the expression levels of miR-101a and miR-30b were detected by qRT-PCR. The results reveal that under
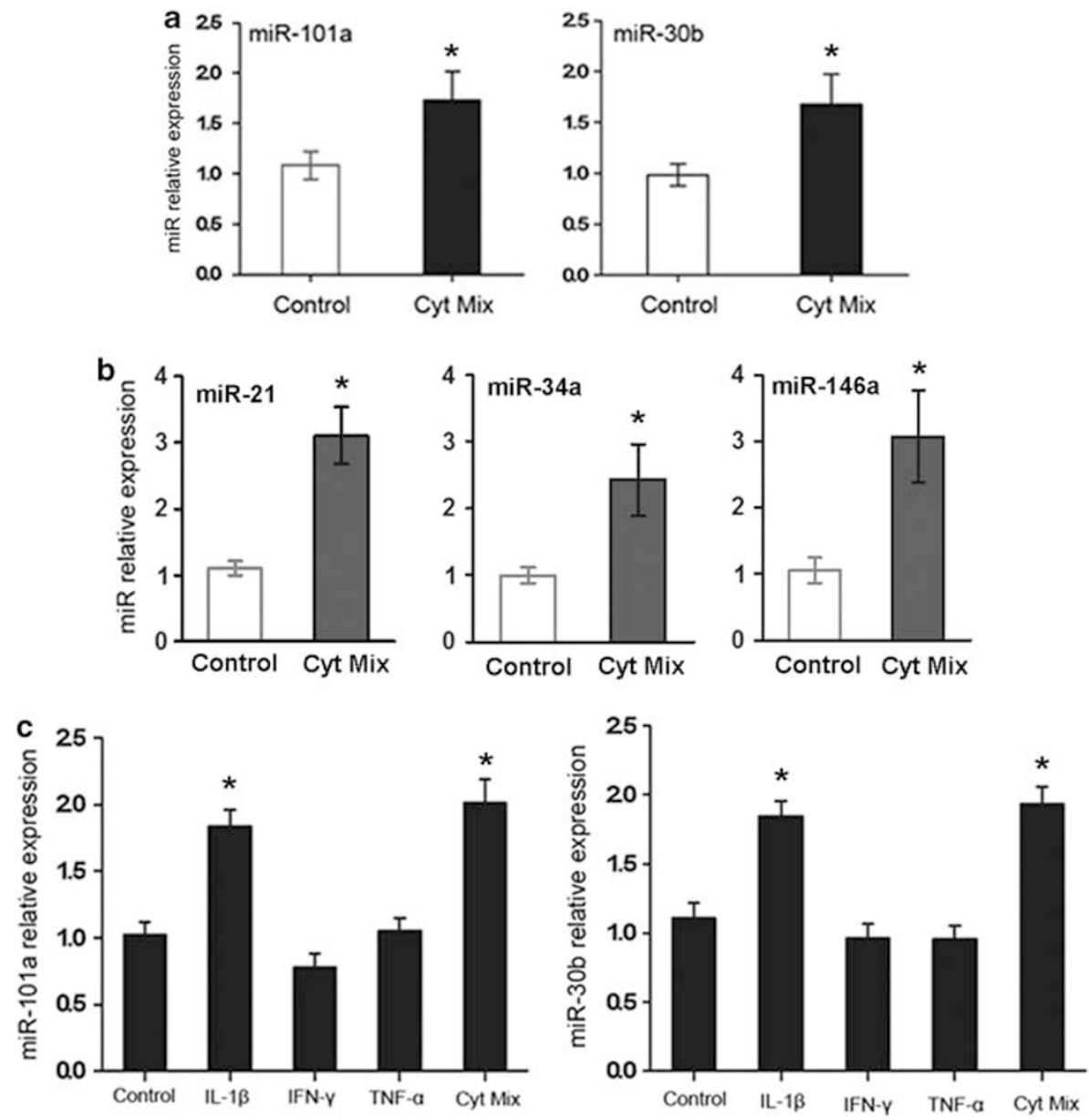

Figure 1 Proinflammatory cytokines increase the expression of miR-101a and miR-30b. (a) MIN6 cells were incubated for $24 \mathrm{~h}$ in the presence or absence of a cytokine mixture (Cyt Mix) of $10 \mathrm{ng} / \mathrm{ml} \mathrm{IL-1} \beta, 10 \mathrm{ng} / \mathrm{ml} \mathrm{TNF}-\alpha$, and $10 \mathrm{ng} / \mathrm{ml} \mathrm{IFN-} \gamma$. The expression of miR-101a (left panel) and miR-30b (right panel) was assessed by real-time PCR. The expression levels are normalized to U6. The results are expressed as mean \pm s.d. for three independent experiments. (b) The expression levels of miR-21, miR-34a, and miR-146a in inflammatory cytokine-treated MIN6 cells were determined by real-time PCR, respectively. The expression levels are normalized to U6. The results are expressed as mean \pm s.d. for three independent experiments. (c) MIN6 cells were incubated in the presence of the indicated cytokines $(10 \mathrm{ng} / \mathrm{ml})$ for $24 \mathrm{~h}$. The expression of miR-101a (left panel) and miR-30b (right panel) was determined by real-time PCR, and normalized to the level of U6. The data are mean \pm s.d. of three independent experiments. ${ }^{*} P<0.05$, compared with respective control. 
cytokine-induced cytotoxic condition, the expression of miR-101a and miR-30b increased (Figure 1a). We also determined the expression levels of miR-21, miR-34a, and miR-146a, which had been confirmed in Roggli E's study. The results show that a mix of inflammatory cytokines actually induced an increase of the three miRNAs (Figure 1b). To
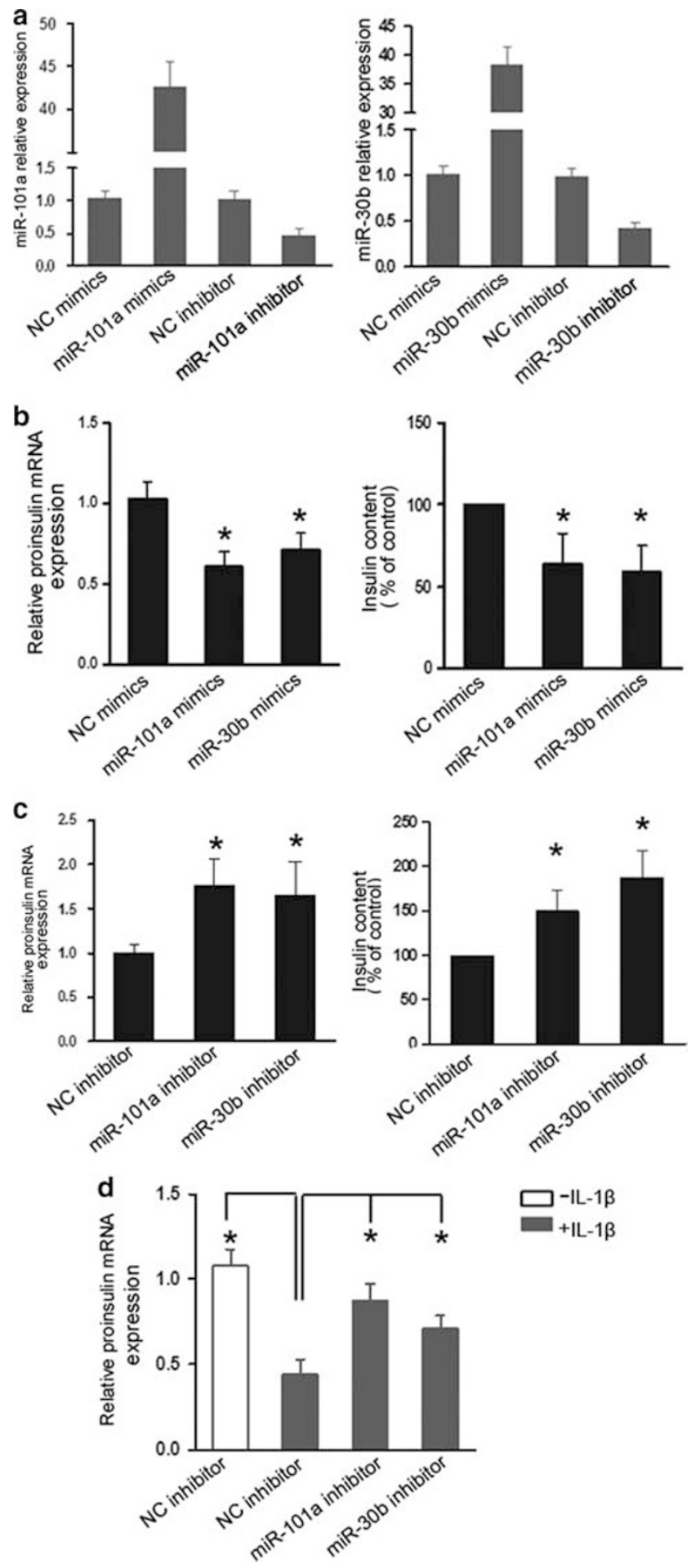

further determine the effects of different cytokines in the induction of miR-101a and miR-30b, the MIN6 cells were treated with $10 \mathrm{ng} / \mathrm{ml} \mathrm{IL-} 1 \beta, 10 \mathrm{ng} / \mathrm{ml}$ TNF- $\alpha, 10 \mathrm{ng} / \mathrm{ml}$ IFN- $\gamma$, or a mix of these cytokines for $24 \mathrm{~h}$. We observed that the two miRNAs expression could be induced by IL-1 $\beta$ treatment, but not by TNF- $\alpha$ or IFN- $\gamma$ (Figure 1b).

\section{miR-101a and miR-30b Impair Insulin Biosynthesis}

To test the effects of miR-101a and miR-30b on pancreatic $\beta$-cell function, we overexpressed or inhibited the two miRNAs by transfecting miRNAs mimics or inhibitors (Figure 2a). We then assessed the impact of the two miRNAs on insulin biosynthesis. The results show that overexpression of miR-101a or miR-30b led to a reduction of proinsulin mRNA levels and total insulin content in MIN6 cells, whereas blockade of endogenous miRNAs function increased proinsulin expression and insulin content (Figures $2 \mathrm{~b}$ and $\mathrm{c}$ ). To further confirm the role of miR-101a and miR-30b in inflammatory cytokine-induced $\beta$-cell dysfunction, the MIN6 cells were transfected with miR-101a or miR-30b inhibitor, or a control antisense oligonucleotide. The day after transfection, the cells were incubated for $24 \mathrm{~h}$ in the presence of $10 \mathrm{ng} / \mathrm{ml}$ IL-1 $\beta$, and the proinsulin mRNA levels were determined by qRT-PCR. As expected, treatment of the cells with IL- $1 \beta$ resulted in a decrease in proinsulin mRNA levels (Figure 2d). However, transfection of the cells with miR-101a or miR-30b inhibitor could partially prevent the decreases in the cells, which indicated that pretreatment of miR-101a or miR-30b inhibitor was able to restore insulin biosynthesis in IL-1 $\beta$ treated cells (Figure 2d). These results suggest that the changes in miR-101a and miR-30b expression mediated by inflammatory cytokines have an important role in the impairment of insulin biosynthesis.

Figure 2 Effect of miR-101a and miR-30b on insulin biosynthesis. (a) The MIN6 cells were transfected with miRNAs mimics or inhibitors. The total RNA was extracted, and the expression levels of miR-101a (left panel) or miR-30b (right panel) were determined to evaluate the efficiency of transfection. The results are mean \pm s.d. of three independent experiments. (b) The MIN6 were transfected with miRNAs mimics or negative control. Proinsulin mRNA levels were measured 2 days later by quantitative RT-PCR, and results are expressed normalized to GAPDH. Insulin content was assessed by ELISA, and the results are expressed as percent of the values in control cells. The data shown are the mean \pm s.d. of three independent experiments. (c) The MIN6 were transfected with miRNAs inhibitors or negative control. Proinsulin mRNA levels and insulin content was assessed by qRT-PCR and ELISA assays. The results are the mean \pm s.d. of three independent experiments. (d) MIN6 cells were transfected with miR-101a or miR-30b inhibitor. The day after, the cells were incubated for $24 \mathrm{~h}$ in the presence or absence of $10 \mathrm{ng} / \mathrm{ml} \mathrm{IL-1 \beta}$. Proinsulin mRNA levels were determined by quantitative RT-PCR. The results shown are the mean \pm s.d. of three independent experiments. ${ }^{*} P<0.05$, compared with respective control. ELISA, enzyme-linked immunosorbent assay; GAPDH, glyceraldehyde-3-phosphate dehydrogenase. 


\section{Pancreas-Specific Transcription Factor Neurod1 Is a Direct Target of miR-101a and miR-30b}

Having established a role for miR-101a and miR-30b in suppressing insulin synthesis in MIN6 cells, we next aimed to clarify its mechanism. We considered it unlikely that

\section{a Position 1227-1234 of Neurod 1 3' UTR 5' UACUGGAAUUGACAUGUACUGUA 3

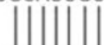 \\ mmu-miR-101a 3' AAGUCAAUAGUGUCAUGACAU 5}

Mutant binding sites $5^{\prime}$ UACUGGAUUGACAUUGACUCGA 3 '

Position 60-67 of Neurod 1 3' UTR 5' GUACAGUGACUGUCCUGUUUACA $3^{\prime}$ mmu-miR-30b 3' UCGACUCACAUCCU--ACAAAUGU 5'

Mutant binding sites 5 ' GUACCUCGACUGUCCCACCGCAC 3 '
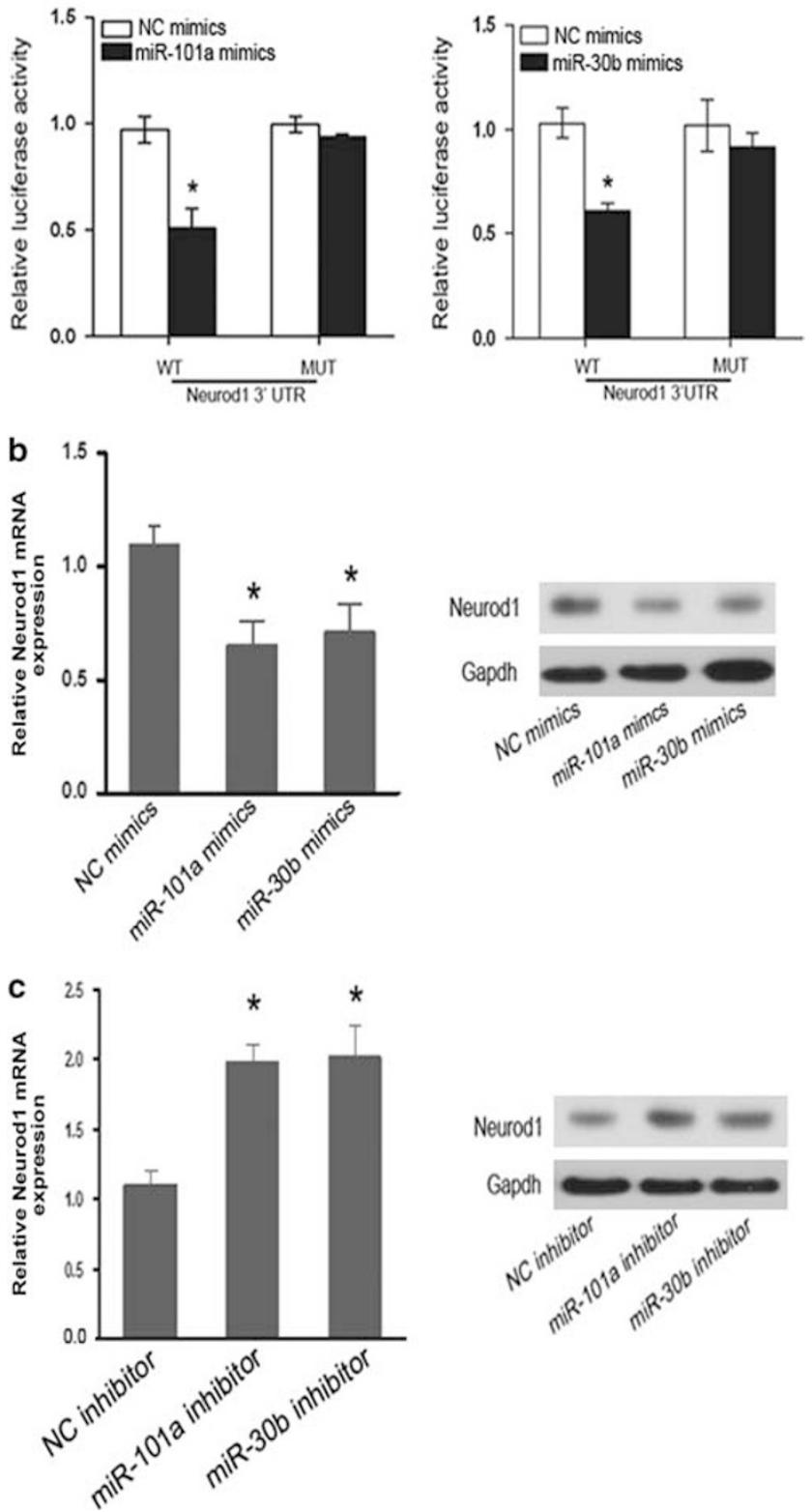

miR-101a and miR-30b target the insulin gene directly because the insulin mRNA 3'-UTR doesn't contain any TargetScan-predicted binding sites. We used the online software TargetScan to predict potential target genes of miR-101a and miR-30b. Surprisingly, the transcriptional factor Neurodl was found to be a predicted target of the two miRNAs. Neurod1 is an important transcriptional activator of insulin gene expression. To obtain direct evidence that Neurodl $3^{\prime}$-UTR is a target of miR-101a and miR-30b, we subcloned the fragments of the Neurod1 $3^{\prime}$-UTR containing the target sequences, or the fragments whose target sites were mutated, into a luciferase reporter vector, respectively. By co-transfection of the reporter plasmids and miR-101a or miR-30b mimics into HEK293 cells, we found that miR-101a and miR-30b mimics markedly decreased the activities of wildtype luciferase reporter vectors, but had no effect on the corresponding mutant luciferase vectors (Figure 3a). Furthermore, transfection of miR-101a or miR-30b mimics decreased Neurod1 expression in MIN6 cells in both the mRNA and protein levels (Figure $3 \mathrm{~b}$ ), whereas transfection of miR-101a or miR-30b inhibitors increased Neurod1 expression (Figure 3c), suggesting that Neurod1 expression could be inhibited by miR-101a and miR-30b via both translational inhibition and mRNA degradation. Taken together, these data demonstrate that miR-101a and miR-30b selectively target Neurod1 and downregulate its expression.

\section{miR-101a but Not miR-30b Suppresses Insulin Release}

We next investigated the effects of miR-101a and miR-30b on the secretory capacity of MIN6 cells. Overexpression of miR-101a did not affect basal secretion but reduced glucoseinduced insulin release (Figure 4a). Nevertheless, miR-30b overexpression had no significant effect on insulin secretion (Figure 4a). To further confirm the effect of miR-101a on insulin release in inflammatory cytokine-mediated $\beta$-cell dysfunction, miR-101a or miR-30b inhibitor was transfected into the cells, and then incubated in the presence of IL- $1 \beta$

Figure 3 Neurod1 is a direct target of miR-101a and miR-30b. (a) miR-101a or miR-30b binds to the $3^{\prime}$-UTR of Neurod1 as shown by the predicted binding of miR-101a or miR-30b. HEK293 cells were cotransfected with luciferase reporter plasmids containing a wild-type (WT) or a mutated (MUT) sequence of the $3^{\prime}$-UTR of mouse Neurod 1 and a renilla transfection control plasmid with miRNA mimics or control mimics. The luciferase reporter assays were performed $48 \mathrm{~h}$ after transfection. The data shown are the mean \pm s.d. of three replicates and are representative of three independent experiments. (b) Overexpression of miR-101a or miR-30b decreases Neurod1 expression. MIN6 cells were transfected with miR-101a or miR-30b mimics, or a negative control. The mRNA and protein expression levels of Neurod1 were determined by qRT-PCR (left panel) and western blot (right panel). (c) MIN6 cells were transiently transfected with a control inhibitor or with miR-101a or miR-30b inhibitor. The expression levels of Neurod1 were analyzed by qRT-PCR (left panel) and western blot (right panel). ${ }^{*} P<0.05$, compared with respective control. GAPDH, glyceraldehyde-3-phosphate dehydrogenase; 3'-UTR, 3'-untranslated region. 

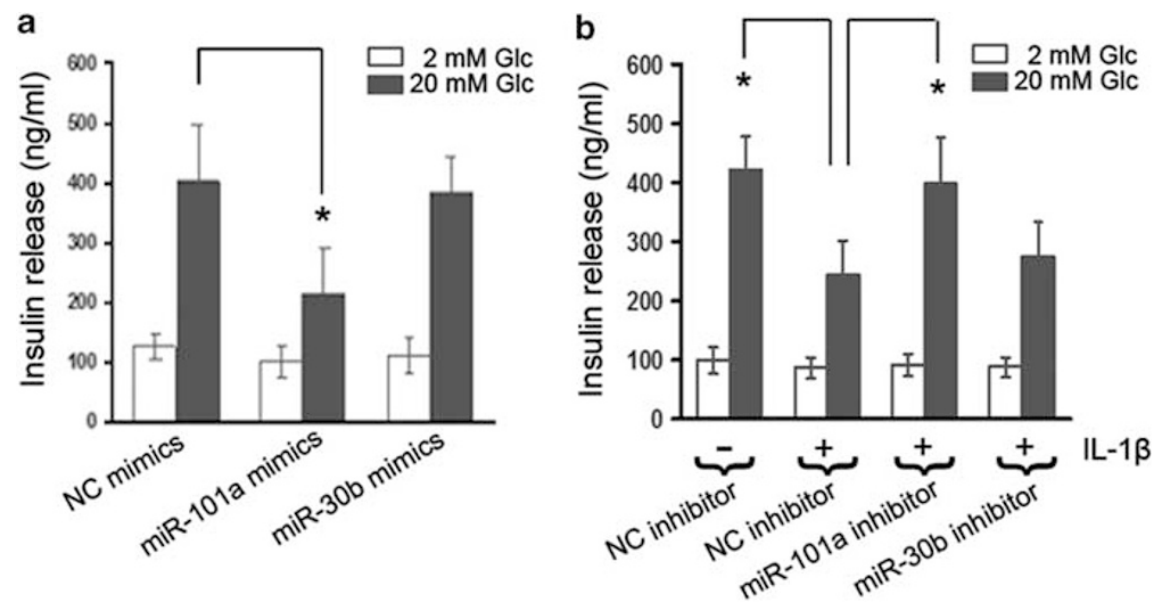

Figure 4 Involvement of miR-101a in insulin secretion. (a) MIN6 cells were transiently transfected with a negative control or the indicated miRNAs. Two days later, the cells were incubated in the presence of 2 or $20 \mathrm{mmol} / \mathrm{l}$ glucose (Glc) for $45 \mathrm{~min}$. The amount of insulin secreted during the incubation period was assessed by ELISA assays. (b) MIN6 cells were transfected with the indicated miRNA inhibitor or a control inhibitor. The day after, they were incubated for $24 \mathrm{~h}$ with or without IL-1 $\beta(2 \mathrm{ng} / \mathrm{ml})$. Insulin release in the presence of 2 and $20 \mathrm{mmol} / \mathrm{l}$ glucose was assessed by ELISA assays. These results shown are the mean \pm s.d. of six replicates and are representative of three independent experiments. ${ }^{*} P<0.05$, compared with respective control. ELISA, enzyme-linked immunosorbent assay.

$(2 \mathrm{ng} / \mathrm{ml})$. After $24 \mathrm{~h}$ stimulation, insulin from supernatants was detected by ELISA assays. The results show that the

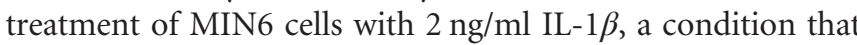
alone did not significantly affect cell survival owing to a lower dose of IL-1 $\beta$ (data not shown), led to impaired GSIS (Figure $4 \mathrm{~b}$ ). However, anti-miR-101a pretreatment was able to alleviate the secretory defect elicited by IL- $1 \beta$ (Figure 4b).

\section{miR-101a Targets Directly Onecut2, Thereby Inhibiting Insulin Secretion}

Insulin release from pancreatic $\beta$-cells has an essential role in blood glucose homeostasis. The transcription factor, Onecut2, has vital roles in regulating insulin release. It is noteworthy that, by using bioinformatic tools, Onecut 2 may be a potential target of both miR-101a and miR-30b. The above results revealed that only miR-101a but not miR-30b can suppress insulin secretion. So, next, we investigated whether Onecut2 is a direct target of miR-101a and miR-30b. The fragments of the Onecut2 3'-UTR containing the target sequences were cloned into downstream of a luciferase reporter vector, and the corresponding pMIR-3'-UTR-MUT was also constructed. The vectors were then co-transfected into the MIN6 cells with miR-101a mimics, miR-30b mimics or negative control. It was found that increased levels of miR-101a inhibited the activity of a luciferase construct containing the $3^{\prime}$-UTR sequence of Onecut2 (Figure 5a), whereas this effect was abrogated by mutation of the sequence recognized by the miRNA, indicating a direct interaction between the $3^{\prime}$-UTR of Onecut 2 and miR-101a. In contrast, miR-30b did not affect the activity, suggesting that only miR-101a is able to recognize the $3^{\prime}$-UTR of the Onecut2 gene (Figure 5a). In agreement with this finding, qRT-PCR and western blot analysis confirmed that overexpression of miR-101a decreased
Onecut2 expression both in mRNA and protein levels, whereas miR-30b did not have effect on Onecut2 expression (Figure 5b). These data reveal that miR-101a targets directly Onecut2, thereby downregulating its expression and suppressing insulin release, whereas miR-30b does not exert the function.

\section{miR-101a and miR-30b Promote Cytokine-Induced $\beta$-Cell Apoptosis}

Prolonged exposure of $\beta$-cells to cytokines may mediate $\beta$-cell apoptosis in autoimmune diabetes. The effect of elevated levels of miR-101a and miR-30b induced by cytokines stimulation on cell survival was next investigated. Overexpression of miR-101a or miR-30b in MIN6 cells caused increased number of cells undergoing apoptosis (Figure 6a). Conversely, transfection of the cells with miR-101a or miR-30b inhibitor diminished the number of dying cells in the presence of a high dose of IL- $1 \beta(10 \mathrm{ng} / \mathrm{ml})$, which is known to cause apoptosis (Figure 6b).

\section{miR-101a and miR-30b Trigger $\beta$-cell Apoptosis by Inhibiting Bcl2}

We postulated that miR-101a and miR-30b may promote apoptosis by suppressing the antiapoptotic protein $\mathrm{Bcl} 2$ in MIN6 cells. Interestingly, the mouse $\mathrm{Bcl} 2$ gene is a potential target of miR-30b as predicted by Targetscan. Therefore, we performed luciferase reporter assays to demonstrate that mmu-miR-30b binds to the $3^{\prime}$-UTR region of $\mathrm{Bcl} 2$ (Figure 7a). Moreover, qRT-PCR and western blot analysis also show that overexpression of miR-30b could downregulate Bcl2 expression levels, whereas transfection of MIN6 cells with miR-30b inhibitor increased Bcl2 expression (Figures $7 \mathrm{~b}$ and c). These data suggest that miR-30b regulates Bcl2 by directly targeting its 3'-UTR. The mouse Bcl2 3'-UTR 
a

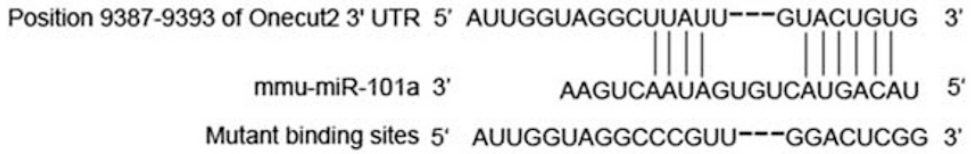

Position 9845-9851 of Onecut2 3' UTR 5' CAGAAUGAGCCCAAAGUUUACAG 3' mmu-miR-30b 3' UCGACUCACAUCCUACAAAUGU 5'

Mutant binding sites 5' CAGAAUGAGCCCAAAUGCAGUCG 3'
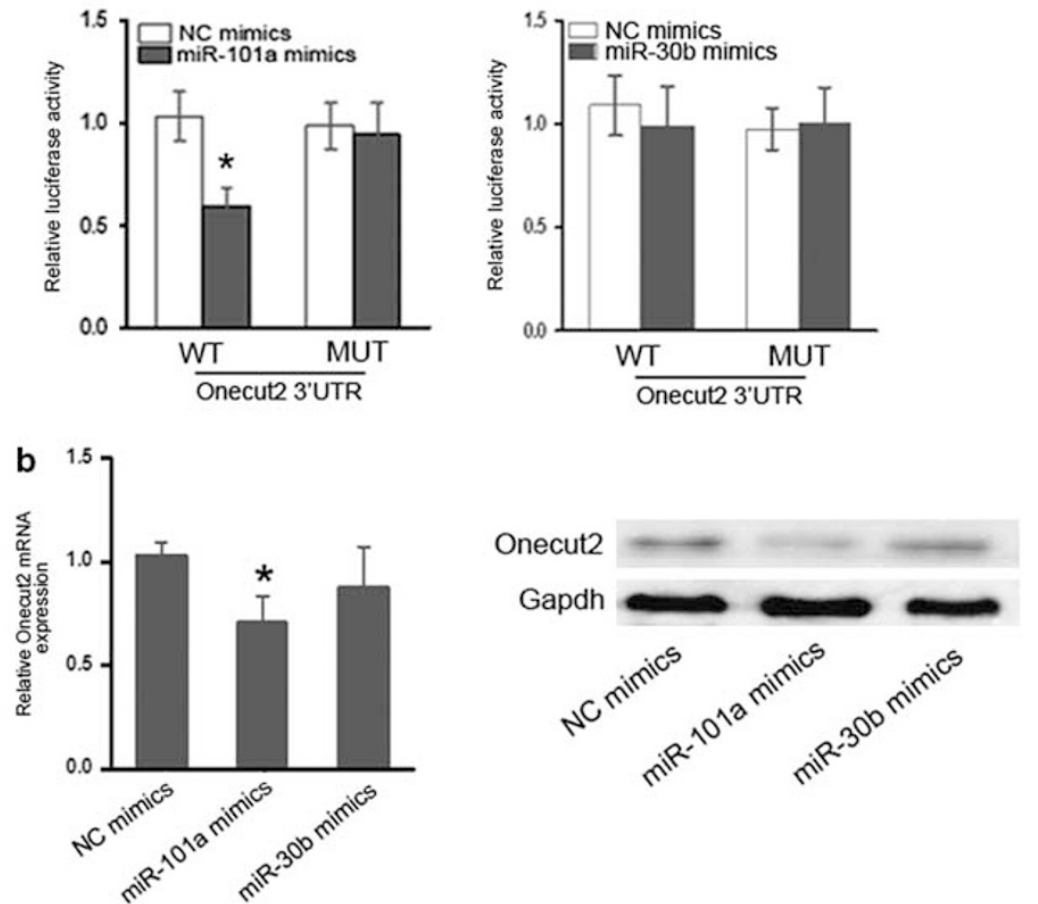

Figure 5 miR-101a binds to Onecut2 3'-UTR and regulates its expression, but miR-30b does not. (a) Potential binding sites of miR-101a and miR-30b within the Onecut2 3'-UTR are shown. HEK293 cells were transiently co-transfected with relative luciferase reporter constructs containing either a wildtype (WT) or a mutated (MUT) Onecut2-binding site sequences with miR-101a or miR-30b mimics. Two days later, luciferase activities were measured and normalized to a renilla luciferase activity. The data shown are the mean \pm s.d. of three replicates and are representative of three independent experiments. (b) Only miR-101a but not miR-30b downregulates Onecut2 expression. A real-time PCR analysis was performed $36 \mathrm{~h}$ after transfection with miR-101a or miR-30b mimics, or with a negative control. A western blot analysis was performed $48 \mathrm{~h}$ after transfection with miR-101a or miR-30b mimics, or with a negative control. ${ }^{*} P<0.05$, compared with respective control. GAPDH, glyceraldehyde-3-phosphate dehydrogenase; 3'-UTR, 3'-untranslated region.

doesn't appear to contain a binding site of miR-101a. It has been reported that hsa-miR-101a may negatively regulate $\mathrm{Bcl} 2$ expression through suppression of STAT3. Next, we investigated whether miR-101a could affect STAT3 expression level, consequently downregulating Bcl2 expression in MIN6 cells. To address this question, MIN6 cells were transfected with miR-101a mimics or a negative control, and then STAT3 and $\mathrm{Bcl} 2$ expression were determined by western blot. The result confirms that there is a significant reduction of the level of STAT3 and Bcl2 in MIN6 cells overexpressing miR-101a (Figure 7d). To demonstrate a direct link between the reduction of STAT3 and the effect of miR-101a on Bcl2 expression, miR-101a was transfected into MIN6 cells with STAT3-expressing vector. As shown in Figure 7e, transfection of the cells with STAT3-expressing vector abrogated the inhibitory effect of miR-101a on Bcl2 expression, indicating that at least part of the effect of miR-101a on Bcl2 expression is mediated by STAT3 pathway.

\section{DISCUSSION}

During the development of type 1 diabetes, inflammatory cytokines are believed to be key mediators of $\beta$-cell dysfunction. $^{2}$ Chronic exposure of $\beta$-cells to inflammatory mediators will result in changes in gene expression, loss of cellular function, and cell death by apoptosis. ${ }^{2-4}$ miRNAs are important regulators of gene expression, and several miRNAs including the miR-29 family and miR-34, have been reported as upregulated by prolonged exposure to cytokines, with 

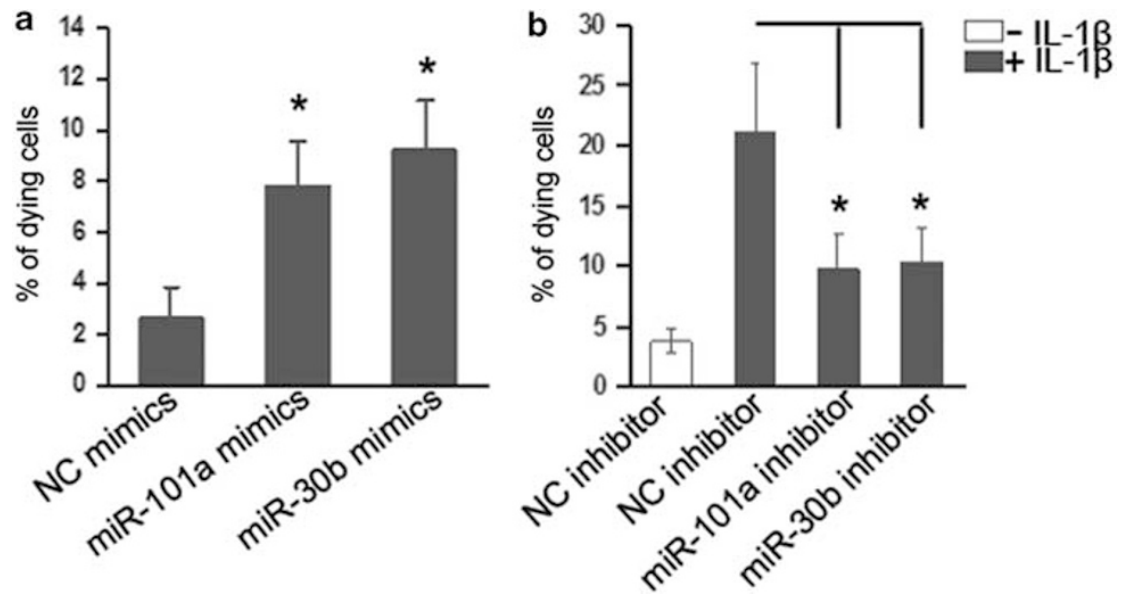

Figure 6 Effect of miR-101a and miR-30b on $\beta$-cell apoptosis. (a) MIN6 cells were transfected with a negative control or miRNA mimics, leading to a rise of the indicated miRNAs. The fraction of dying cells was determined 3 days later by scoring the cells displaying picnotic nuclei upon Hoechst 33342 staining. (b) MIN6 cells were transfected with a control oligonucleotide or miRNAs inhibitor. Two days later, the cells were incubated for $24 \mathrm{~h}$ with or without $10 \mathrm{ng} /$ $\mathrm{ml} \mathrm{IL-1 \beta}$. Dying cells were scored as described above. Results are the mean of three experiments. ${ }^{*} P<0.05$, compared with respective control.

effects on the secretory capacities of $\beta$-cells and their sensitivity to apoptosis. ${ }^{14,15}$

In the present study, we demonstrate two additional miRNAs that are induced in cytokine-treated $\beta$-cells, miR-101a, and miR-30b. These two miRNAs, which are expressed in mouse pancreatic $\beta$-cells, are increased after treatment with a cytokine mix. Furthermore, expression of the miRNAs is induced by IL- $1 \beta$ treatment, but not by TNF- $\alpha$ or IFN- $\gamma$. These results are in good agreement with those obtained previously by Roggli's microarray analyses. ${ }^{14}$ IL- $1 \beta$ has been proposed to have a key role in inflammatory cytokine-mediated destruction of pancreatic $\beta$-cells during the development of autoimmune type 1 diabetes. IL- $1 \beta$ has a more pronounced pro-apoptotic effect than TNF- $\alpha$ or IFN- $\gamma .{ }^{16}$ The exposure of $\beta$-cells to IL- $1 \beta$ alone results in impairment of $\beta$-cell specialized function, but not TNF- $\alpha$ or IFN- $\gamma$. IL- $1 \beta$ causes the $\beta$-cell damage mainly by regulating cellular signaling pathways such as Fas, NF- $\kappa \mathrm{B}$ and an others. ${ }^{17,18}$ Previous studies demonstrated that expression of miR-101a is modulated by the PI3K/Akt signaling pathway, which is activated in response to IL- $1 \beta \cdot{ }^{19,20}$ Wei et al. ${ }^{21}$ have demonstrated that miR-30b is positively regulated by NF- $\kappa B$. Therefore, we believe that IL- $1 \beta$ alone is capable of inducing alterations in miR-101a and miR-30b expression, and that IL-1 $\beta$-mediated signaling pathways participate in the induction of miR-101a and miR-30b in $\beta$-cells, though the latter hypothesis requires further investigation.

Our results demonstrate that miR-101a and miR-30b downregulate proinsulin expression and decrease total insulin content in MIN6 cells. Moreover, blockade of endogenous miR-101a and miR-30b can restore insulin biosynthesis in IL- $1 \beta$-treated $\beta$-cells. These data suggest that miR-101a and miR-30b may contribute to an impairment of insulin biosynthesis mediated by inflammatory cytokines. Insulin gene transcription is tightly regulated by a network of transcriptional activators and repressors that maintain $\beta$-cell fate and function. ${ }^{22}$ Transcriptional activators, such as Pdx1, MafA, and Neurod1, facilitate insulin transcription, ${ }^{23-25}$ whereas transcriptional repressors of the insulin gene, including Hes1, Sox6, and Crem, have the opposite effect. ${ }^{26-28}$ Neurod1 has been shown to be upregulated in tandem under high-glucose conditions, which can bind to the E element of the insulin genes and modulate the expression of genes. ${ }^{24}$ The transcription factor Neurod1 is a predicted target of miR-101a and miR-30b. In agreement with the computational predictions, our study shows that miR-101a and miR-30b negatively regulate Neurod 1 expression through direct base pairing with its $3^{\prime}$-UTR. In brief, miR-101a and miR-30b have a key role in a defective insulin production during cytokine-mediated $\beta$-cell failure.

Insulin release from pancreatic $\beta$-cells is a precisely regulated event permitting tight control of blood glucose levels. Several proteins controlling insulin exocytosis have been identified, but the factors determining the expression of the components of the secretory machinery in $\beta$-cells remain largely unknown. Herein, we demonstrate that miR-101a has a critical role in the control of the secretory function of insulin-producing cells. Overexpression of miR-101a leads to a loss in the efficiency of the cells to respond to glucose stimuli, whereas pretreatment with miR-101a inhibitor could improve GSIS in cytokine-treated cells. We also examined the effect of miR-30b on GSIS, but no effect was observed, in agreement with Plaisance's previous study. ${ }^{9}$ miR-101a suppresses GSIS in pancreatic $\beta$-cells, probably via an alteration in the components of the secretory machinery and their regulatory proteins. Several proteins controlling insulin exocytosis have been reported, including Rab3, Rab27 GTPases, and their effectors Noc2 and Granuphilin. ${ }^{29,30}$ Granuphilin is a Rab3/Rab27 effector that is associated with dense-core secretory granules of pancreatic $\beta$-cells and few other endocrine cells. ${ }^{9}$ Granuphilin exerts a potent negative modulatory action on insulin 
exocytosis, and maintaining adequate levels of this protein appears to be a critical prerequisite for preserving insulin secretory capacity. ${ }^{9}$ Onecut2, a key transcription factor, controls the expression of granuphilin, a Rab guanine triphosphate (GTP)ase effector that colocalizes with the dense-core, insulin-containing granules of pancreatic $\beta$-cells and acts as a potent inhibitor of insulin exocytosis. ${ }^{9}$ In the current study, Onecut2 was confirmed to be a direct target of miR-101a by luciferase reporter assays. qRT-PCR and western blot analysis also revealed a decrease in the level of the transcriptional factor Onecut 2 upon miR-101a overexpression. Thus, by decreasing
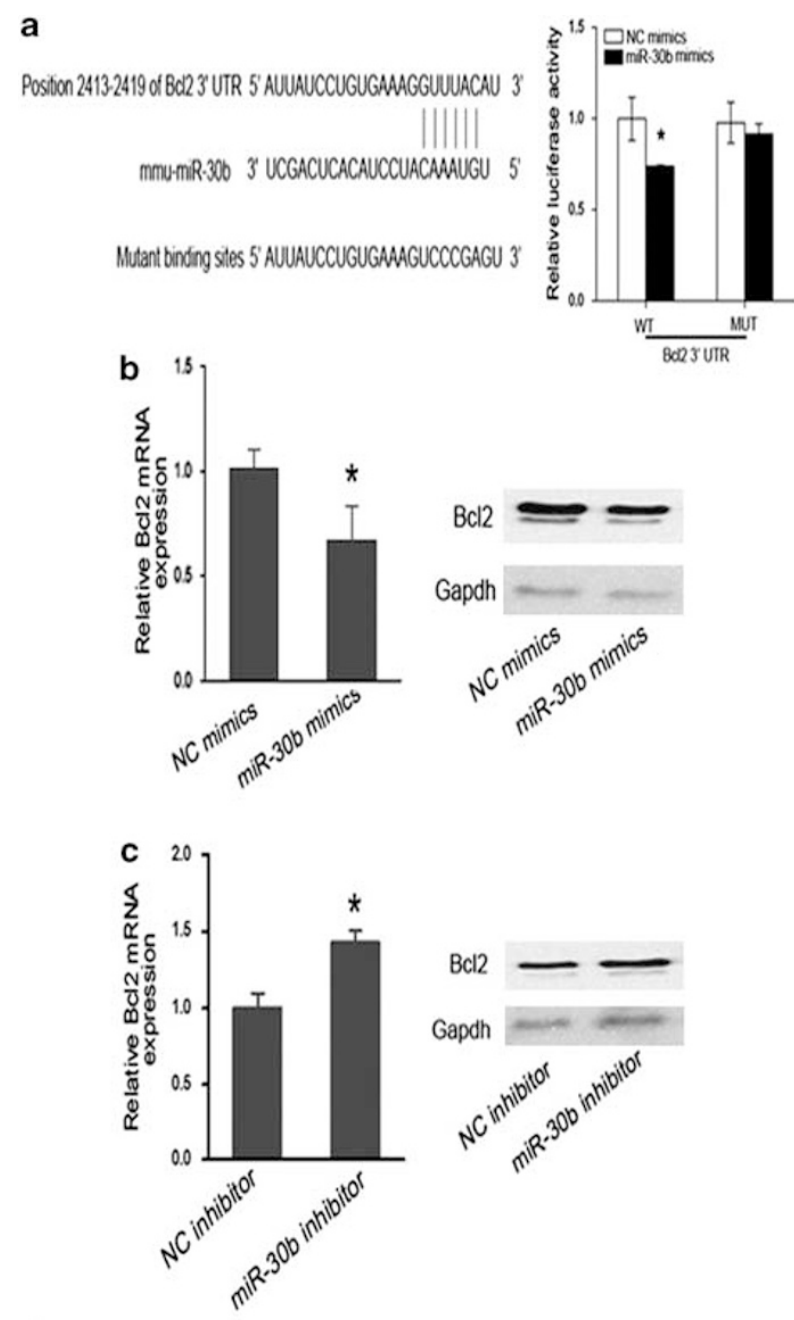

d

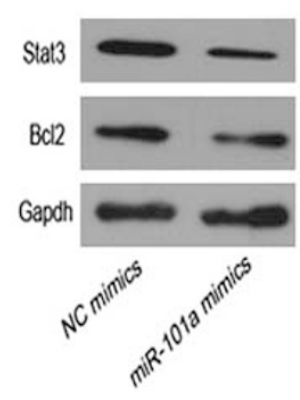

Onecut2 levels, miR-101a is likely to enable increased amounts of granuphilin, resulting in a defective insulin secretion.

Prolonged exposure to proinflammatory cytokines is known to affect $\beta$-cell survival. ${ }^{2,4}$ However, the exact mechanism underlying the cytotoxic effects of the inflammatory cytokines is not completely understood. In our study, we demonstrated that overexpression of miR-101a and miR-30b in MIN6 cells increases apoptosis, whereas blockade of the two miRNAs protects $\beta$-cells from the deleterious effect of inflammatory mediators. Thus, miR-101a and miR-30b may participate in the process of cytokine-mediated $\beta$-cell apoptosis. Inflammatory cytokines can trigger $\beta$-cell death through the 'mitochondrial' or intrinsic pathway of apoptosis. This pathway is regulated by the Bcl-2 (B-cell lymphoma-2) protein family, which includes pro- (such as the Bcl-2 homology 3 (BH3)-only proteins) and antiapoptotic proteins, which mainly include Bcl-2, Bcl-XL, myeloid cell leukemia sequence-1 (Mcl-1) and A1 (also known as Bcl-2A1). ${ }^{31-34}$ $\mathrm{Bcl} 2$, a member of antiapoptosis protein family, has been shown to protect $\beta$-cells from cytokine-induced cell death, which can control mitochondrial integrity, and the cysteinylaspartate-specific proteases or caspases, thereby inhibiting apoptosis. $^{31}$ Hsa-miR-101a and hsa-miR-30b have been determined to reduce $\mathrm{Bcl} 2$, consequently accelerating apoptosis. $^{21,35}$ Therefore, we assumed that the effect of miR-101a and miR-30b on apoptosis may be also associated with the antiapoptotic protein $\mathrm{Bcl} 2$ in MIN6 cells. By luciferase reporter assays, we confirmed that mmu-miR-30b can bind to $3^{\prime}$-UTR of mouse $\mathrm{Bcl} 2$ gene, as predicted by TargetScan. qRT-PCR and western blot analysis also showed that miR-30b reduces Bcl 2 expression in MIN6 cells. As for miR-101a, no recognition sites in $3^{\prime}$-UTR region of $\mathrm{Bcl} 2$ mRNA are identified, which suggests an indirect effect of the miRNA on $\mathrm{Bcl} 2$ expression. Koukus et al. ${ }^{35}$ reported that miR-101a could regulate $\mathrm{Bcl} 2$ through the STAT3 pathway. Whether a similar mechanism is operating in $\beta$-cells remains

Figure 7 Impact of miR-101a and miR-30b on $\mathrm{Bcl} 2$ expression. (a) miR-30b binds to the $3^{\prime}$-UTR of $\mathrm{Bcl} 2$ as shown by the predicted binding site. miR-30b regulates the expression of the Bcl2 $3^{\prime}$-UTR reporter constructs. The luciferase reporter assays were performed $48 \mathrm{~h}$ after transfection with the indicated pMIR-REPORT plasmids with miR-30b mimics or a negative control. (b) Overexpression of miR-30b decreases $\mathrm{Bcl} 2$ expression. MIN6 cells were transfected with miR-30b mimics or a negative control. The mRNA and protein expression levels of $\mathrm{Bcl} 2$ were determined by qRT-PCR (left panel) and western blot (right panel). (c) MIN6 cells were transiently transfected with a control inhibitor or miR-30b inhibitor. The expression levels of Bcl2 were analyzed by qRT-PCR (left panel) and western blot (right panel). (d) MIN6 cells were transfected with miR-101a mimics or control mimics. After $48 \mathrm{~h}$, total proteins were extracted, and the STAT3 and BCl2 protein levels were determined by western blot. (e) STAT3-overexpressing vector was transfected into MIN6 cells with miR-101a mimics or a negative control. And then the $\mathrm{Bcl} 2$ protein levels were determined by western blot. ${ }^{*} P<0.05$, compared with respective control. GAPDH, glyceraldehyde-3-phosphate dehydrogenase; MUT, mutated; WT, wild type; 3'-UTR, 3'-untranslated region. 
to be established. We observed that overexpression of miR-101a reduces the expression of Bcl2 in MIN6 cells, whereas transfection of the cells with STAT3-expressing vector could abrogate the effect, indicating that miR-101a may decrease $\mathrm{Bcl} 2$ expression at least partly by suppressing STAT3 pathway in MIN6 cells. These results suggest that miR-101a and miR-30b can directly or indirectly suppress the antiapoptotic protein, Bcl2. Given that downregulation of this antiapoptotic protein is a crucial event, leading to $\beta$-cell apoptosis in response to cytokines, we believe that miR-101a and miR-30b have a vital role in cytokine-mediated $\beta$-cell death and may also serve as therapeutic strategies to selectively promote survival for $\beta$-cells in autoimmune type 1 diabetes.

Although the insulin therapy has improved the lives of people affected by autoimmune type 1 diabetes, it is still one of the most challenging health problems worldwide, owing to high rates of complications in type 1 diabetes. A number of studies showed that miRNAs have an important role in the etiology and pathogenesis of type 1 diabetes and its complications. Changes in miRNA expression in type 1 diabetes cause $\beta$-cell dysfunction and promote cell apoptosis. Restoration of expression of miRNAs to normal levels may act as a potential therapy for maintaining sufficient insulin secretion. Our findings suggest that miR-101a and miR-30b significantly inhibit insulin production and promote $\beta$-cell apoptosis, by targeting some related genes. Considering that human miR-101a and miR-30b genes share similar sequences with mouse miR-101a and miR-30b, they may have a pivotal role in $\beta$-cell dysfunction in NOD mouse and human islets, thus leading to the onset and development of type 1 diabetes. In light of the important role of miR-101a and miR-30b in type 1 diabetes, they may become novel pharmacological targets for the treatment of type 1 diabetes in the future.

Because the current study was carried out in vitro, there are questions that need to be addressed in the future. Studies in NOD mice, an animal model sharing many characteristics of human type 1 diabetes, may enhance our understanding of the contribution of miR-101a and miR-30b to the development of this disease in vivo. It will also be important to confirm the roles of miR-101a and miR-30b during the initial phases of type 1 diabetes in humans. Nonetheless, this study provides evidence that the contributions of miR-101a and miR-30b to $\beta$-cell dysfunction are associated with their regulation of relative function genes. Because each miRNA can regulate the expression of many mRNAs, the identification of additional miR-101a and miR-30b targets promises to highlight new candidate genes potentially involved in type 1 diabetes.

\section{ACKNOWLEDGMENTS}

This work was supported by National Natural Science Foundation of China (81300648, 81200644, 81170725), Hunan Province Natural Sciences

Foundation of China (15JJ3132).

\section{DISCLOSURE/CONFLICT OF INTEREST}

The authors declare no conflict of interest.

1. Zhang L, Gianani R, Nakayama $M$ et al. Type 1 diabetes: chronic progressive autoimmune disease. Novartis Found Symp 2008;292:85-94.

2. Eizirik DL, Colli ML, Ortis F. The role of inflammation in insulitis and beta-cell loss in type 1 diabetes. Nat Rev Endocrinol 2009;5:219-226.

3. McDaniel ML, Kwon G, Hill JR et al. Cytokines and nitric oxide in islet inflammation and diabetes. Proc Soc Exp Biol Med 1996;211:24-32.

4. Donath MY, Storling J, Berchtold LA et al. Cytokines and beta-cell biology: from concept to clinical translation. Endocr Rev 2008;29:334-350.

5. Bartel DP. Micrornas: genomics, biogenesis, mechanism, and function. Cell 2004;116:281-297.

6. Guo H, Ingolia NT, Weissman JS et al. Mammalian micrornas predominantly act to decrease target mrna levels. Nature 2010;466: 835-840.

7. Chang TC, Mendell JT. Micrornas in vertebrate physiology and human disease. Annu Rev Genomics Hum Genet 2007;8:215-239.

8. Poy MN, Eliasson L, Krutzfeldt J et al. A pancreatic islet-specific microrna regulates insulin secretion. Nature 2004;432:226-230.

9. Plaisance V, Abderrahmani A, Perret-Menoud $V$ et al. Microrna-9 controls the expression of granuphilin/slp4 and the secretory response of insulin-producing cells. J Biol Chem 2006;281:26932-26942.

10. Baroukh N, Ravier MA, Loder MK et al. Microrna-124a regulates Foxa2 expression and intracellular signaling in pancreatic beta-cell lines. J Biol Chem 2007;282:19575-19588.

11. Lovis P, Gattesco S, Regazzi R. Regulation of the expression of components of the exocytotic machinery of insulin-secreting cells by micrornas. Biol Chem 2008;389:305-312.

12. El Ouaamari A, Baroukh N, Martens GA et al. Mir-375 targets 3'phosphoinositide-dependent protein kinase- 1 and regulates glucoseinduced biological responses in pancreatic beta-cells. Diabetes 2008;57:2708-2717.

13. Lovis $\mathrm{P}$, Roggli $\mathrm{E}$, Laybutt $\mathrm{DR}$ et al. Alterations in microrna expression contribute to fatty acid-induced pancreatic beta-cell dysfunction. Diabetes 2008;57:2728-2736.

14. Roggli E, Britan A, Gattesco S et al. Involvement of micrornas in the cytotoxic effects exerted by proinflammatory cytokines on pancreatic beta-cells. Diabetes 2010;59:978-986.

15. Roggli E, Gattesco S, Caille D et al. Changes in microrna expression contribute to pancreatic beta-cell dysfunction in prediabetic Nod mice. Diabetes 2012;61:1742-1751.

16. Ortis F, Miani M, Colli ML et al. Differential usage of Nf-Kappa $b$ activating signals by II-1beta and Tnf-alpha in pancreatic beta cells. FEBS Lett 2012;586:984-989.

17. Loweth AC, Williams GT, James RF et al. Human islets of langerhans express fas ligand and undergo apoptosis in response to interleukin1 beta and Fas ligation. Diabetes 1998;47:727-732.

18. Flodstrom M, Welsh N, Eizirik DL. Cytokines activate the nuclear factor kappa B (Nf-Kappa B) and induce nitric oxide production in human pancreatic islets. FEBS Lett 1996;385:4-6.

19. Yang J, Lu YW, Lu MM et al. Microrna-101, mitogen-activated protein kinases and mitogen-activated protein kinases phosphatase-1 in systemic lupus erythematosus. Lupus 2013;22:115-120.

20. Zhu QY, Liu Q, Chen JX et al. Microrna-101 targets Mapk phosphatase1 to regulate the activation of Mapks in macrophages. J Immunol 2010;185:7435-7442.

21. Wei C, Li L, Gupta S. Nf-kappab-mediated Mir-30b regulation in cardiomyocytes cell death by targeting Bcl-2. Mol Cell Biochem 2014;387:135-141.

22. Andrali SS, Sampley ML, Vanderford NL et al. Glucose regulation of insulin gene expression in pancreatic beta-cells. Biochem J 2008;415:1-10.

23. Petersen HV, Serup P, Leonard J et al. Transcriptional regulation of the human insulin gene is dependent on the homeodomain protein Stf1/ Ipf1 acting through the Ct boxes. Proc Natl Acad Sci USA 1994;91: 10465-10469.

24. Naya FJ, Stellrecht CM, Tsai MJ. Tissue-specific regulation of the insulin gene by a novel basic helix-loop-helix transcription factor. Genes Dev 1995;9:1009-1019.

25. Kaneto H, Miyatsuka T, Kawamori D et al. Pdx-1 and Mafa play a crucial role in pancreatic beta-cell differentiation and maintenance of mature beta-cell funcation. Endocr J 2008;55:235-252. 
26. Iguchi $\mathrm{H}$, Ikeda $\mathrm{Y}$, Okamura $\mathrm{M}$ et al. Sox6 attenuates glucose-stimulated insulin secretion by repressing pdx 1 transcriptional activity and is down-regulated in hyperinsulinemic obese mice. J Biol Chem 2005;280:37669-37680.

27. Inada A, Yamada $Y$, Someya $Y$ et al. Transcriptional repressors are increased in pancreatic islets of type 2 diabetic rats. Biochem Biophys Res Commun 1998;253:712-718.

28. Jensen J, Pedersen EE, Galante $P$ et al. Control of endodermal endocrine development by Hes-1. Nat Genet 2000;24:36-44.

29. Cheviet $S$, Waselle $L$, Regazzi R. Noc-king out exocrine and endocrine secretion. Trends Cell Biol 2004;14:525-528.

30. Lang J. Molecular mechanisms and regulation of insulin exocytosis as a paradigm of endocrine secretion. Eur J Biochem 1999;259:3-17.
31. Youle RJ, Strasser A. The Bcl-2 protein family: opposing activities that mediate cell death. Nat Rev Mol Cell Biol 2008;9:47-59.

32. Gurzov EN, Ortis F, Cunha DA et al. Signaling by Il-1beta+Ifn-gamma and Er stress converge on Dp5/Hrk activation: a novel mechanism for pancreatic beta-cell apoptosis. Cell Death Differ 2009;16:1539-1550.

33. Gurzov EN, Eizirik DL. Bcl-2 proteins in diabetes: mitochondrial pathways of beta-cell death and dysfunction. Trends Cell Biol 2011;21:424-431.

34. Akerfeldt MC, Howes J, Chan JY et al. Cytokine-induced beta-cell death is independent of endoplasmic reticulum stress signaling. Diabetes 2008;57:3034-3044.

35. Koukos G, Polytarchou C, Kaplan JL et al. Microrna-124 regulates Stat3 expression and Is down-regulated in colon tissues of pediatric patients with ulcerative colitis. Gastroenterology 2013;145:842-852. 